\title{
Oncolytic immunotherapy for metastatic cancer: lessons and future strategies
}

\author{
Zong-Sheng Guo \\ UPMC Hillman Cancer Center and Department of Surgery, University of Pittsburgh School of Medicine, Pittsburgh, PA, USA \\ Correspondence to: Zong-Sheng Guo, PhD. UPMC Hillman Cancer Center and Department of Surgery, University of Pittsburgh School of Medicine, \\ 5117 Centre Avenue, Pittsburgh, PA 15213, USA. Email: guoxzs@upmc.edu. \\ Provenance and peer review: This article was commissioned and reviewed by the editorial office, Annals of Translational Medicine. The article did not \\ undergo external peer review. \\ Comment on: Schenk EL, Mandrekar SJ, Dy GK, et al. A Randomized Double-Blind Phase II Study of the Seneca Valley Virus (NTX-010) versus \\ Placebo for Patients with Extensive-Stage SCLC (ES SCLC) Who Were Stable or Responding after at Least Four Cycles of Platinum-Based \\ Chemotherapy: North Central Cancer Treatment Group (Alliance) N0923 Study. J Thorac Oncol 2020;15:110-9.
}

Submitted Mar 23, 2020. Accepted for publication Apr 03, 2020.

doi: $10.21037 / \mathrm{atm} .2020 .04 .42$

View this article at: http://dx.doi.org/10.21037/atm.2020.04.42

Schenk et al. conducted a randomized, double-blind and placebo-controlled phase II study with oncolytic picornavirus NTX-010 for extensive-stage small-cell lung cancer and produced negative results. These results could be attributed mainly to low viral dose and a more advanced stage of metastatic cancer treated, and the oncolytic virus used belonged to the first generation with minimal potency to elicit antitumor immunity. I envision that in the future, oncolytic immunotherapy will achieve much improved therapeutic efficacy in patients with ES-SCLC or other metastatic cancers by utilizing better armed OVs and rational combination strategies. These combinations with other immunotherapeutic regimens, immunogenic chemotherapy, radiation and/or targeted agents can promote potent antitumor immunity and other tumoricidal mechanisms.

\section{Introduction}

"Ninety nine percent of success is built on failure."-By Charles F. Kettering.

The approval of the oncolytic virus (OV) T-VEC (Imlygic ${ }^{\mathrm{TM}}$ ) for the treatment of advanced melanoma by the US and Europe in 2015 showcased the promise of this novel type of cancer immunotherapy (1-3). Since then, the exciting therapeutics of this has been moving forward rapidly, however, many more basic and clinical studies need to be conducted and several major issues need to be addressed before this therapeutic modality can evolve into a standard care for a variety of cancer patients.

Small-cell lung cancer (SCLC) accounts for $\sim 15 \%$ of all lung malignancies. This is a type of neuroendocrine tumor with highly aggressive behavior, characterized by rapid growth and tendency for widespread metastasis. When diagnosed, over $70 \%$ of new SCLC patients represent stage IV disease. In these patients, clinical onset often correlates with a heavy symptomatic burden and rapid decline of overall health (4). Over the past two decades, very little progress has been made; thus, chemotherapy and radiotherapy remain the mainstay of treatment for SCLC. Yet, recurrence happens early and often, leading to a dismal prognosis and a 2.8\% 5-year overall survival for the extensive-stage disease. Therefore, novel and more effective strategies to treat SCLC are badly needed. Immune checkpoint inhibitors (ICIs) have been tested in multiple clinical studies but so far clinical efficacy has been limited. Various forms of immunotherapy for SCLC represent a promising approach. However, a number of challenges lie ahead (4).

\section{Brief history of Senecavirus as an OV}

Senecavirus A, formerly known as Seneca Valley virus, is a non-enveloped RNA virus of the genus Senecavirus, 
family Picornaviridae. It was accidently discovered by Genetic Therapy Inc. as contaminant in a human cell culture in 2002, and designated as Seneca Valley Virus isolate 001 (SVV-001; now called NTX-010) (5). In 2007, the first two studies investigating this virus in tumor cells in vitro and in tumor models in vivo generated important discoveries. In the first study (6), the authors found that, first, neuroendocrine and pediatric tumor cells are sensitive, at least 10,000-fold more sensitive to the cytotoxicity of this picornavirus than any adult normal human cells tested. Second, they also found that the viral infectivity was not inhibited by human blood components, suggesting that this OV could be delivered intravenously. Indeed, in tumorbearing immunocompetent mice, intravenous doses of up to $1.0 \mathrm{e} 14$ viral particles (vp) were well tolerated and no toxicity was observed. In the second study, another group of authors also found no toxicity, but high efficacy in an invasive and metastatic retinoblastoma model treated with systemic delivery of a dose at $1.0 \mathrm{e} 13 \mathrm{vp} / \mathrm{kg}$ (7). In 2010, another group of authors examined the infectivity, cytotoxicity, and efficacy in human cancer cells in vitro and in xenografts in vivo (8). They found a marked cytotoxicity in a subset of the cell lines from the panel of neuroblastoma, Ewing sarcoma, and rhabdomyosarcomas. In vivo the most consistent efficacy was observed for the rhabdomyosarcoma and neuroblastoma panels. Finally, Liu et al. studied its toxicity and efficacy in an orthotopic xenograft model of pediatric glioma (9). They found that this OV exhibits strong antitumoral activity in pediatric glioma and it utilizes $\alpha 2,3$-linked and $\alpha 2,6$-linked sialic acids as mediators of tumor cell infection (9). All these preclinical studies set up a solid foundation for clinical trials with this nonpathogenic OV.

Two phase I studies were conducted and published. Rudin and colleagues evaluated the $\mathrm{OV}$ in patients with cancers with neuroendocrine features by single intravenous doses across five log-increments from $10^{7}$ to $10^{11} \mathrm{vp} / \mathrm{kg}(10)$. Even the highest dose of the virus was well tolerated, with predictable virus clearance kinetics, viral replication in the tumor, and some evidence of antitumoral activity in SCLC. In the other study, Burke and colleagues have tested this OV in children with relapsed/refractory solid tumors (11). NTX-010 was tolerable, even at the highest dose tested either alone or in combination with cyclophosphamide. However, the addition of cyclophosphamide did not prevent the production of neutralizing antibodies and thus the virus got cleared rapidly. As for efficacy, no objective responses were observed at the time of disease re-evaluation, even though stable disease was observed in $6 / 12$ patients treated with the virus and 4/6 patients in combination with cyclophosphamide (11). These studies warranted a phase II clinical evaluation in SCLC.

\section{Summary of results}

In this randomized, double-blind and placebo-controlled phase II clinical study (12), a total of 50 patients with ES SCLC in whom the disease did not progress after four or more cycles of platinum-based chemotherapy were randomized 1:1 to receive a single dose of NTX-010 $(n=26)$ or placebo $(n=24)$ within 12 weeks of chemotherapy. NTX010 or placebo was infused intravenously as a $1 \mathrm{~h}$ infusion in $100 \mathrm{~mL}$ normal saline as a single dose. The primary end point was progression-free survival (PFS). Viral clearance and detection of neutralizing antibodies were followed. At the specified interim analysis, the median PFS was 1.7 months (95\% CI: $1.4-3.1$ months) for the NTX-010 group versus 1.7 months (95\% CI: $1.4-4.3$ months) for the placebo group. The trial was terminated owing to futility.

The measurements of viral clearance and neutralizing antiviral antibodies were followed to explore if longer viral exposure had any association with improved clinical outcomes. The neutralizing antibodies were detected on day 14 after viral treatment in all 23 evaluable patients. Viral clearance was observed in most of the 26 patients who received NTX-010 either seven or 14 days after administration. Several patients had persistent virus. Most investigators would interpret the detectable levels of virus at later times as secondary to intratumoral viral replication and a potential marker of efficacy. In contradiction, in this study, delayed clearance of the virus was associated with shorter PFS and overall survival (OS). My personal point of view is that these patients had more immunosuppression, and more advanced cancer, were less likely to generate antiviral immunity (and antitumor immunity), and thus more likely to have viral replication in cancer tissues, leading to more persistence of the virus in the body. Indeed, persistent viremia could be a negative biomarker for patients' response to this treatment.

\section{Discussion and commentary}

To examine the reasons behind this negative study (12), we need to analyze the type of cancer patients, viral dose and methodologies used in this trial, and assess if the underlying hypothesis was robust. To obtain fair assessments, we 
must rely on our current understanding of oncolytic immunotherapy and status of the type of cancer and connect this trial to other similar clinical studies and findings. The main reasons for negative results are: (I) a first generation, not-so-efficacious OV and the use of NTX-010 at a dose much lower than that used in preclinical studies; (II) an aggressive type of cancer; (III) a single agent that cannot elicit potent and sustained antitumor immunity in order to achieve effective therapy. Unfortunately, in this and other clinical and preclinical studies with NTX-010, very little, if any, immunological endpoints have been analyzed. The lessons learned from this study will be discussed in more detail, and future strategies will be followed.

\section{The viral dose and type of human SCLC were no match}

In previous preclinical studies, the authors showed that intravenous injection of up to $1.0 \mathrm{e} 13$ or $1.0 \mathrm{e} 14 \mathrm{vp} / \mathrm{kg}$ led to good therapeutic results in invasive retinoblastoma or neuroendocrine tumor models, respectively $(6,7)$. In the two previous clinical studies, little efficacy was observed at the maximal administered viral doses $(10,11)$, and that predicted the outcome of the phase II study. In this phase II study, the viral dose was $1.0 \mathrm{e} 11 \mathrm{vp} / \mathrm{kg}$. This is 100 or 1,000 -fold lower than that used in the preclinical studies and was meant to treat a very fast-growing and aggressive cancer, an extensive stage of SCLC. These two factors, lower viral dosage and the highly aggressive and extensive-stage of the disease made the clinical trial destined for failure.

\section{Better armed OVs for enhanced antitumor immunity}

The OV used in this study, NTX-010, is a nonrecombinant, replication competent oncolytic RNA virus. In other words, it is a first generation OV without any exogenous genes to promote its efficacy. OV armed with weak immunostimulatory gene may work in immunogenic tumors. For example, GM-CSF-armed T-VEC worked to a degree in advanced melanoma, presumably the most immunogenic type of cancer. After treatment with T-VEC, the median OS was 23.3 months as compared to 18.9 months with GM-CSF, an extension of 4.4 months (1). Another similarly GM-CSF-armed oncolytic poxvirus, called Pexa-Vec, failed in a 2019 Phase III trial assessing its combination with Nexavar ${ }^{\circledR}$ (sorafenib) compared to Nexavar alone in advanced liver cancer. Advanced liver cancer is lowly immunogenic and highly immunosuppressive: thus, it is very difficult to achieve positive clinical responses with a relatively weak $\mathrm{OV}$ in this type of cancer.

Many preclinical studies have explored whether OVs armed with more potent Th1-cytokines for improved antitumor immunity $(3,13,14)$, such as OVs expressing genetically engineered IL-2, IL-15 or an inducible T cell co-stimulator (ICOS) could promote systemic antitumor immunity (15-17). It is envisioned that the new generation of armed OVs would be more efficacious in cancer patients.

Immunotherapy works most efficiently against highly immunogenic cancers, and cold tumors are a therapeutic challenge for immunotherapy (18). That is why many studies of cancer immunotherapy have been initially conducted with melanoma, one of the highly immunogenic type of cancers. T-VEC (armed with GM-CSF) was barely effective in advanced melanoma. All considered, the second lesson is that we should not set the aim too high by treating most advanced cancers using just a plain OV (alone). SCLCs show reasonable levels of tumor mutational burden, and good quantities of neoantigens targets for antitumor immunity (19). Therefore, SCLC, in theory, is suitable disease for immunotherapy. However, it is one of the very aggressive diseases, and the oncolysis potency of this weak OV, without much help from a potent antitumor immunity, could not get the job done.

\section{Combination regimens as rational strategies}

We and many others believe that OVs provide an ideal platform for combination therapy $(20,21)$. Combining an $\mathrm{OV}$ and immune checkpoint blockade has proven to be a highly promising strategy. Preclinical studies showed that OVs often induce inflammation, leading to the expression of checkpoint molecules such as PD-L1 in tumor and turn cold tumor hot, making the strategy of combining an $\mathrm{OV}$ with immune checkpoint blockade a rational strategy (22). In melanoma patients, the combination improved the objective clinical response rates from $20 \%$ in monotherapy to about $65 \%$ when combined $(23,24)$. Therefore, it would be interesting to study this combination strategy in cold tumors with low tumor mutational burden such as pancreatic cancers (25).

Clinical data on the efficacy of monotherapy with ICIs are not so promising despite a sound biological background for SCLC (4). However, rational combinations make sense. One obvious combination strategy would be to combine $\mathrm{OV}$ with ICI as OV may induce inflammation as well as antitumor immunity, leading to upregulation of PD-1/ 
PD-L1 and possibly other immune checkpoint molecules, targets for ICIs (22-24). Other combination strategies with OVs may include, but are not limited to, cytotoxic chemotherapy, targeted agents, radiation therapy, and other forms of immunotherapy such as cytokines, adoptive $\mathrm{T}$ cell therapy and other forms of cancer vaccines.

\section{Perspectives}

Cancer immunotherapy for SCLC has a solid biological rationale, but so far treatments with either ICIs or OVs have shown little therapeutic effects. Based on our current understanding of cancer biology, immunology, pharmacology and virology, I believe that oncolytic immunotherapy should be combined with other regimens of immunotherapy, immunogenic chemotherapy, radiation or/ and targeted agents in order to optimize tumor cell killing, modulate the tumor microenvironment, and elicit potent and sustained antitumor immunity to achieve significantly improved clinical responses. With further preclinical and clinical studies using these rational combination strategies, we will achieve much better efficacy for ES-SCLC and other types of metastatic cancers.

\section{Acknowledgments}

I thank Ms. Christine Burr for manuscript editing. Funding: None.

\section{Footnote}

Conflicts of Interest: The author has completed the ICMJE uniform disclosure form (available at http://dx.doi. org/10.21037/atm.2020.04.42). The author has a patent "Oncolytic virus therapy" pending and a patent "Oncolytic virus expressing IL-36 gamma" pending.

Ethical Statement: The author is accountable for all aspects of the work in ensuring that questions related to the accuracy or integrity of any part of the work are appropriately investigated and resolved.

Open Access Statement: This is an Open Access article distributed in accordance with the Creative Commons Attribution-NonCommercial-NoDerivs 4.0 International License (CC BY-NC-ND 4.0), which permits the noncommercial replication and distribution of the article with the strict proviso that no changes or edits are made and the original work is properly cited (including links to both the formal publication through the relevant DOI and the license). See: https://creativecommons.org/licenses/by-nc-nd/4.0/.

\section{References}

1. Andtbacka RH, Kaufman HL, Collichio F, et al. Talimogene laherparepvec improves durable response rate in patients with advanced melanoma. J Clin Oncol 2015;33:2780-8.

2. Guo ZS. The 2018 Nobel Prize in medicine goes to cancer immunotherapy (editorial for BMC cancer). BMC Cancer 2018;18:1086.

3. Harrington K, Freeman DJ, Kelly B, et al. Optimizing oncolytic virotherapy in cancer treatment. Nat Rev Drug Discov 2019;18:689-706.

4. Pavan A, Attili I, Pasello G, et al. Immunotherapy in small-cell lung cancer: from molecular promises to clinical challenges. J Immunother Cancer 2019;7:205.

5. Burke MJ. Oncolytic Seneca Valley Virus: past perspectives and future directions. Oncolytic Virother 2016;5:81-9.

6. Reddy PS, Burroughs KD, Hales LM, et al. Seneca Valley virus, a systemically deliverable oncolytic picornavirus, and the treatment of neuroendocrine cancers. J Natl Cancer Inst 2007;99:1623-33.

7. Wadhwa L, Hurwitz MY, Chevez-Barrios P, et al. Treatment of invasive retinoblastoma in a murine model using an oncolytic picornavirus. Cancer Res 2007;67:10653-6.

8. Morton CL, Houghton PJ, Kolb EA, et al. Initial testing of the replication competent Seneca Valley virus (NTX010) by the pediatric preclinical testing program. Pediatr Blood Cancer 2010;55:295-303.

9. Liu Z, Zhao X, Mao H, et al. Intravenous injection of oncolytic picornavirus SVV-001 prolongs animal survival in a panel of primary tumor-based orthotopic xenograft mouse models of pediatric glioma. Neuro Oncol 2013;15:1173-85.

10. Rudin CM, Poirier JT, Senzer NN, et al. Phase I clinical study of Seneca Valley Virus (SVV-001), a replicationcompetent picornavirus, in advanced solid tumors with neuroendocrine features. Clin Cancer Res 2011;17:888-95.

11. Burke MJ, Ahern C, Weigel BJ, et al. Phase I trial of Seneca Valley Virus (NTX-010) in children with relapsed/ refractory solid tumors: a report of the Children's Oncology Group. Pediatr Blood Cancer 2015;62:743-50.

12. Schenk EL, Mandrekar SJ, Dy GK, et al. A Randomized Double-Blind Phase II Study of the Seneca Valley 
Virus (NTX-010) versus Placebo for Patients with Extensive-Stage SCLC (ES SCLC) Who Were Stable or Responding after at Least Four Cycles of Platinum-Based Chemotherapy: North Central Cancer Treatment Group (Alliance) N0923 Study. J Thorac Oncol 2020;15:110-9.

13. Bartlett DL, Liu Z, Sathaiah M, et al. Oncolytic viruses as therapeutic cancer vaccines. Mol Cancer 2013;12:103.

14. Pearl TM, Markert JM, Cassady KA, et al. Oncolytic Virus-Based Cytokine Expression to Improve Immune Activity in Brain and Solid Tumors. Mol Ther Oncolytics 2019;13:14-21.

15. Zamarin D, Holmgaard RB, Ricca J, et al. Intratumoral modulation of the inducible co-stimulator ICOS by recombinant oncolytic virus promotes systemic antitumour immunity. Nat Commun 2017;8:14340.

16. Kowalsky SJ, Liu Z, Feist M, et al. Superagonist IL15-Armed Oncolytic Virus Elicits Potent Antitumor Immunity and Therapy That Are Enhanced with PD-1 Blockade. Mol Ther 2018;26:2476-86.

17. Liu Z, Ge Y, Wang H, et al. Modifying the cancer-immune set point using vaccinia virus expressing re-designed interleukin-2. Nat Commun 2018;9:4682.

18. Bonaventura P, Shekarian T, Alcazer V, et al. Cold Tumors: A Therapeutic Challenge for Immunotherapy. Front Immunol 2019;10:168.

19. Alexandrov LB, Ju YS, Haase K, et al. Mutational

Cite this article as: Guo ZS. Oncolytic immunotherapy for metastatic cancer: lessons and future strategies. Ann Transl Med 2020;8(17):1113. doi: 10.21037/atm.2020.04.42 signatures associated with tobacco smoking in human cancer. Science 2016;354:618-22.

20. Guo ZS, Bartlett DL. Oncolytic viruses as platform for multimodal cancer therapeutics: a promising land. Cancer Gene Ther 2014;21:261-3.

21. Bommareddy PK, Shettigar M, Kaufman HL. Integrating oncolytic viruses in combination cancer immunotherapy. Nat Rev Immunol 2018;18:498-513.

22. Liu Z, Ravindranathan R, Kalinski P, et al. Rational combination of oncolytic vaccinia virus and PD-L1 blockade works synergistically to enhance therapeutic efficacy. Nat Commun 2017;8:14754.

23. Ribas A, Dummer R, Puzanov I, et al. Oncolytic Virotherapy Promotes Intratumoral T Cell Infiltration and Improves Anti-PD-1 Immunotherapy. Cell 2017;170:110919 e10.

24. Chesney J, Puzanov I, Collichio F, et al. Randomized, Open-Label Phase II Study Evaluating the Efficacy and Safety of Talimogene Laherparepvec in Combination With Ipilimumab Versus Ipilimumab Alone in Patients With Advanced, Unresectable Melanoma. J Clin Oncol 2018;36:1658-67.

25. Yarchoan M, Hopkins A, Jaffee EM. Tumor Mutational Burden and Response Rate to PD-1 Inhibition. N Engl J Med 2017;377:2500-1. 